\title{
Noninvasive characterization of tumor angiogenesis and oxygenation in bevacizumab-treated recurrent glioblastoma by using dynamic susceptibility MRI: secondary analysis of the European Organization for Research and Treatment of Cancer 26101 Trial.
}

Kickingereder, Philipp ; Brugnara, Gianluca ; Hansen, Mikkel Bo ; Nowosielski, Martha ; Pflüger, Irada ; Schell, Marianne ; Isensee, Fabian ; Foltyn, Martha ; Neuberger, Ulf ; Kessler, Tobias ; Sahm, Felix ; Wick, Antje ; Heiland, Sabine ; Weller, Michael ; Platten, Michael ; von Deimling, Andreas ; Maier-Hein, Klaus H ; Østergaard, Leif ; van den Bent, Martin J ; Gorlia, Thierry ; Wick, Wolfgang ; Bendszus, Martin

\begin{abstract}
Background Relevance of antiangiogenic treatment with bevacizumab in patients with glioblastoma is controversial because progression-free survival benefit did not translate into an overall survival (OS) benefit in randomized phase III trials. Purpose To perform longitudinal characterization of intratumoral angiogenesis and oxygenation by using dynamic susceptibility contrast agent-enhanced (DSC) MRI and evaluate its potential for predicting outcome from administration of bevacizumab. Materials and Methods In this secondary analysis of the prospective randomized phase II/III European Organization for Research and Treatment of Cancer 26101 trial conducted between October 2011 and December 2015 in 596 patients with first recurrence of glioblastoma, the subset of patients with availability of anatomic MRI and DSC MRI at baseline and first follow-up was analyzed. Patients were allocated into those administered bevacizumab (hereafter, the BEV group; either bevacizumab monotherapy or bevacizumab with lomustine) and those not administered bevacizumab (hereafter, the non-BEV group with lomustine monotherapy). Contrast-enhanced tumor volume, noncontrast-enhanced T2 fluid-attenuated inversion recovery (FLAIR) signal abnormality volume, Gaussian-normalized relative cerebral blood volume (nrCBV), Gaussian-normalized relative blood flow (nrCBF), and tumor metabolic rate of oxygen $\left(\mathrm{nTMRO}_{2}\right)$ was quantified. The predictive ability of these imaging parameters was assessed with multivariable Cox regression and formal interaction testing. Results A total of 254 of 596 patients were evaluated (mean age, 57 years $\pm 11 ; 155$ men; 161 in the BEV group and 93 in non-BEV group). Progression-free survival was longer in the BEV group (3.7 months; $95 \%$ confidence interval [CI]: 3.0, 4.2) compared with the non-BEV group (2.5 months; $95 \% \mathrm{CI}: 1.5,2.9 ; \mathrm{P}=.01)$, whereas OS was not different $(\mathrm{P}=.15)$. The nrCBV decreased for the BEV group (-16.3\%; interquartile range [IQR], $-39.5 \%$ to $12.0 \% ; \mathrm{P}=.01)$, but not for the non-BEV group $(1.2 \%$; IQR, $-17.9 \%$ to $23.3 \%$; $=.19)$ between baseline and first follow-up. An identical pattern was observed for both $\mathrm{nrCBF}$ and $\mathrm{nTMRO} \mathrm{Tal}_{2}$ values. Contrast-enhanced tumor and noncontrast-enhanced T2 FLAIR signal abnormality volumes decreased for the BEV group (-66\% [IQR, $-83 \%$ to $-35 \%$ ] and $-33 \%$ [IQR, $-71 \%$ to $-5 \%$ ], respectively; $\mathrm{P}<.001$ for both), whereas they increased for the non-BEV group (30\% [IQR, $-17 \%$ to $98 \%$ ], $\mathrm{P}=.001$; and $10 \%$ [IQR, $-13 \%$ to $82 \%$ ], $\mathrm{P}=.02$, respectively) between baseline and first follow-up. None of the assessed MRI parameters were predictive for $\mathrm{OS}$ in the BEV group. Conclusion Bevacizumab treatment decreased tumor volumes, angiogenesis, and oxygenation, thereby reflecting its effectiveness for extending progression-free survival; however, these parameters were not predictive of overall survival (OS), which highlighted the challenges of identifying patients that derive an OS benefit from bevacizumab. (C) RSNA, 2020 Online supplemental material is available for this article. See also the editorial by Dillon in this issue.
\end{abstract}


Posted at the Zurich Open Repository and Archive, University of Zurich

ZORA URL: https://doi.org/10.5167/uzh-191407

Journal Article

Published Version

Originally published at:

Kickingereder, Philipp; Brugnara, Gianluca; Hansen, Mikkel Bo; Nowosielski, Martha; Pflüger, Irada; Schell, Marianne; Isensee, Fabian; Foltyn, Martha; Neuberger, Ulf; Kessler, Tobias; Sahm, Felix; Wick, Antje; Heiland, Sabine; Weller, Michael; Platten, Michael; von Deimling, Andreas; Maier-Hein, Klaus H; Østergaard, Leif; van den Bent, Martin J; Gorlia, Thierry; Wick, Wolfgang; Bendszus, Martin (2020). Noninvasive characterization of tumor angiogenesis and oxygenation in bevacizumab-treated recurrent glioblastoma by using dynamic susceptibility MRI: secondary analysis of the European Organization for Research and Treatment of Cancer 26101 Trial. Radiology, 297(1):164-175.

DOI: https://doi.org/10.1148/radiol.2020200978 


\title{
Noninvasive Characterization of Tumor Angiogenesis and Oxygenation in Bevacizumab-treated Recurrent Glioblastoma by Using Dynamic Susceptibility MRI: Secondary Analysis of the European Organization for Research and Treatment of Cancer 26101 Trial
}

\author{
Philipp Kickingereder, MD, MBA • Gianluca Brugnara, MD • Mikkel Bo Hansen, PhD • Martha Nowosielski, MD, PhD • \\ Irada Pflüger, MD • Marianne Schell, MD • Fabian Isensee, PhD • Martha Foltyn, MD • Ulf Neuberger, MD • \\ Tobias Kessler, MD • Felix Sahm, MD • Antje Wick, MD • Sabine Heiland, PhD • Michael Weller, MD • \\ Michael Platten, $M D \bullet$ Andreas von Deimling, MD • Klaus H. Maier-Hein, PhD • Leif Østergaard, MD, PhD • \\ Martin J. van den Bent, MD • Thierry Gorlia, PhD • Wolfgang Wick, MD • Martin Bendszus, MD
}

From the Department of Neuroradiology, Heidelberg University Hospital, Im Neuenheimer Feld 400, 69120 Heidelberg, Germany (P.K., G.B., I.P., M.S., M.F.,
U.N., S.H., M.B.); Center of Functionally Integrative Neuroscience and MINDLab, Aarhus University Hospital, Aarhus, Denmark (M.B.H., L.Ø.); Neurology Clinic,
Heidelberg University Hospital, Heidelberg, Germany (M.N., T.K., A.W., W.W.); Department of Neurology, Medical University Innsbruck, Innsbruck, Austria (M.N.);
Medical Image Computing, German Cancer Research Center (DKFZ), Heidelberg, Germany (F.I., K.H.M.H.); Clinical Cooperation Unit Neurooncology, German
Cancer Research Center (DKFZ), Heidelberg, Germany (T.K., W.W.); Department of Neuropathology, Institute of Pathology, Heidelberg University Hospital, Heidel-
berg, Germany (F.S., A.v.D.); Clinical Cooperation Unit Neuropathology, German Cancer Research Center (DKFZ), Heidelberg, Germany (F.S., A.v.D.); Department of
Neurology, University Hospital and University of Zurich, Zurich, Switzerland (M.W.); Department of Neurology, Medical Faculty Mannheim, MCTN, University of Hei-
delberg, Mannheim, Germany (M.P.); Pattern Analysis and Learning Group, Department of Radiation Oncology, Heidelberg University Hospital, Heidelberg, Germany
(K.H.M.H.); Department of Neuroradiology, Aarhus University Hospital, Aarhus, Denmark (L.Ø.); Brain Tumor Center at Erasmus MC Cancer Institute, Rotterdam,
the Netherlands (M.J.v.d.B.); and European Organization for Research and Treatment of Cancer (EORTC), Brussels, Belgium (T.G.). Received March 13, 2020; revision
requested May 4; revision received May 8; accepted May 26. Address correspondence to P.K. (e-mail: philipp.kickingereder@med.uni-heidelberg.de). Supported by the Else-Kröner Memorial Scholarship of the Else Kröner-Fresenius Foundation and the Deutsche Forschungsgemeinschaft (DFG, German Research Foundation) (Project-ID 404521405, SFB 1389-UNITE Glioblastoma, Work Package C02 and Priority Programme 2177 "Radiomics: Next Generation of Biomedical Imaging).

Conflicts of interest are listed at the end of this article.

See also the editorial by Dillon in this issue.

Radiology 2020; 297:164-175 • https://doi.org/10.1148/radiol.2020200978 • Content codes: NR MR

Background: Relevance of antiangiogenic treatment with bevacizumab in patients with glioblastoma is controversial because progression-free survival benefit did not translate into an overall survival (OS) benefit in randomized phase III trials.

Purpose: To perform longitudinal characterization of intratumoral angiogenesis and oxygenation by using dynamic susceptibility contrast agent-enhanced (DSC) MRI and evaluate its potential for predicting outcome from administration of bevacizumab.

Materials and Methods: In this secondary analysis of the prospective randomized phase II/III European Organization for Research and Treatment of Cancer 26101 trial conducted between October 2011 and December 2015 in 596 patients with first recurrence of glioblastoma, the subset of patients with availability of anatomic MRI and DSC MRI at baseline and first follow-up was analyzed. Patients were allocated into those administered bevacizumab (hereafter, the BEV group; either bevacizumab monotherapy or bevacizumab with lomustine) and those not administered bevacizumab (hereafter, the non-BEV group with lomustine monotherapy). Contrast-enhanced tumor volume, noncontrast-enhanced T2 fluid-attenuated inversion recovery (FLAIR) signal abnormality volume, Gaussian-normalized relative cerebral blood volume (nrCBV), Gaussian-normalized relative blood flow (nrCBF), and tumor metabolic rate of oxygen $\left(\mathrm{nTMRO}_{2}\right)$ was quantified. The predictive ability of these imaging parameters was assessed with multivariable Cox regression and formal interaction testing.

Results: A total of 254 of 596 patients were evaluated (mean age, 57 years $\pm 11 ; 155$ men; 161 in the BEV group and 93 in nonBEV group). Progression-free survival was longer in the BEV group (3.7 months; $95 \%$ confidence interval [CI]: 3.0, 4.2) compared with the non-BEV group (2.5 months; $95 \% \mathrm{CI}: 1.5,2.9 ; P=.01)$, whereas OS was not different $(P=.15)$. The nrCBV decreased for the BEV group ( $-16.3 \%$; interquartile range [IQR], $-39.5 \%$ to $12.0 \% ; P=.01)$, but not for the non-BEV group (1.2\%; IQR, $-17.9 \%$ to $23.3 \% ; P=.19$ ) between baseline and first follow-up. An identical pattern was observed for both nrCBF and nTMRO values. Contrast-enhanced tumor and noncontrast-enhanced T2 FLAIR signal abnormality volumes decreased for the BEV group $(-66 \%[\mathrm{IQR},-83 \%$ to $-35 \%]$ and $-33 \%$ [IQR, $-71 \%$ to $-5 \%]$, respectively; $P<.001$ for both), whereas they increased for the non-BEV group (30\% [IQR, $-17 \%$ to $98 \%], P=.001$; and $10 \%$ [IQR, $-13 \%$ to $82 \%$ ], $P=.02$, respectively) between baseline and first follow-up. None of the assessed MRI parameters were predictive for OS in the BEV group.

Conclusion: Bevacizumab treatment decreased tumor volumes, angiogenesis, and oxygenation, thereby reflecting its effectiveness for extending progression-free survival; however, these parameters were not predictive of overall survival (OS), which highlighted the challenges of identifying patients that derive an OS benefit from bevacizumab.

(C) RSNA, 2020

Online supplemental material is available for this article. 


\section{Abbreviations}

$\mathrm{CI}=$ confidence interval, DSC $=$ dynamic susceptibility contrast enhanced, EORTC = European Organization for Research and Treatment of Cancer, FLAIR = fluid-attenuated inversion recovery, IQR = interquartile range, $\mathrm{nrCBF}=$ Gaussian-normalized relative cerebral blood flow, $\mathrm{nrCBV}=$ Gaussian-normalized relative cerebral blood volume, nTMRO ${ }_{2}=$ Gaussian-normalized tumor metabolic rate of oxygen, OS $=$ overall survival

\section{Summary}

This secondary analysis of European Organization for Research and Treatment of Cancer 26101 highlights the biological activity and effectiveness of bevacizumab for extending progression-free survival for patients with glioblastoma; however, identifying patients with overall survival benefit remains challenging.

\section{Key Results}

- Compared with lomustine monotherapy, antiangiogenic treatment for glioblastoma with bevacizumab led to a reduction in tumor volumes, angiogenesis, and oxygenation $(P<.001$ for each).

- Baseline or change in tumor angiogenesis and oxygenation were not associated with overall patient survival after bevacizumab treatment $(P=.59-.95)$.

G lioblastoma is the most common malignant primary brain tumor and, despite decades of research, remains among the most lethal of all forms of cancer. These tumors are highly angiogenic and characterized by vascular proliferations that are substantially fueled by vascular endothelial growth factor signaling. The U.S. Food and Drug Administration approved the use of bevacizumab, a recombinant humanized monoclonal antibody that binds to vascular endothelial growth factor, in patients with recurrent glioblastoma after a number of encouraging (but uncontrolled) phase II trials with promising response rates on MRI scans and delayed tumor progression $(1,2)$. Subsequent randomized phase III trials (Avastin in Glioblastoma, or AVAglio; Radiation Therapy Oncology Group, or RTOG, 0825; and European Organization for Research and Treatment of Cancer [EORTC] 26101 [3-5]) confirmed the significantly longer progression-free survival with the use of bevacizumab; however, this did not translate into an overall survival (OS) benefit, neither in the setting of newly diagnosed tumors when adding bevacizumab to standard treatment nor at first recurrence when comparing bevacizumab with lomustine chemotherapy. Despite the strong biologic rationale for the use of antiangiogenic agents in glioblastoma, the results from the available randomized phase III trials indicate that bevacizumab may not be beneficial in unselected populations of patients with glioblastoma (6). Consequently, there has been a strong interest in identifying predictive biomarkers for selecting patient subsets that are most likely to benefit from bevacizumab $(7,8)$. Specifically, assessment of the tumor vasculature through dynamic susceptibility-weighted contrast agent-enhanced (DSC) MRI may offer a biologically meaningful surrogate for predicting treatment response to antiangiogenic agents (9-11).

We conducted an exploratory secondary analysis of the randomized controlled multicenter phase II and III EORTC 26101 trial with in-depth longitudinal characterization of intratumoral angiogenesis at DSC MRI including quantification of blood volume, blood flow, and metabolic rate of oxygen. Moreover, we investigated the utility of these angiogenesis-related parameters from DSC MRI alongside standard imaging parameters, such as tumor volumes, from anatomic MRI for identifying subsets of patients who are most likely to benefit from bevacizumab by means of OS.

\section{Materials and Methods}

\section{Study Design and Participants}

In this secondary analysis, we analyzed MRI data from patients with brain tumors that were acquired as part of the prospective EORTC 26101 trial (NCT01290939), which was conducted between October 2011 and December 2015. Our study was performed in accordance with the Declaration of Helsinki, and the protocol was approved by local ethics committees and patients provided written informed consent (EudraCT 2010 023218-30). The full study design and outcomes (progressionfree survival and OS) were published previously $(12,13)$.

As described previously (14), EORTC 26101 was a prospective randomized phase II and III trial in patients with first progression of glioblastoma after standard chemotherapy and radiation therapy. In brief, the phase II trial evaluated the optimal treatment sequence of bevacizumab and lomustine (four treatment arms with single agent vs sequential vs combination), whereas the subsequent phase III trial (two treatment arms) compared patients treated with lomustine alone with patients who were administered a combination of lomustine and bevacizumab (randomized at a 1:2 ratio). Overall, EORTC 26101 included 596 patients (159 patients from phase II and 437 patients from phase III) from 37 institutions within Europe. On the basis of the four treatment arms of the phase II trial (12) and the two treatment arms in the phase III trial (13), patients were allocated to this secondary analysis into two groups: (a) those administered bevacizumab (hereafter, referred to as the BEV group) and (b) those not administered bevacizumab (hereafter, referred to as the non-BEV group) for the treatment at first recurrence of glioblastoma.

For our analysis, patients with availability of MRI that included anatomic sequences (pre- and postcontrast T1-weighted, T2-weighted, and fluid-attenuated inversion recovery [FLAIR] images) and DSC MRI sequences at baseline before treatment and at first follow-up were selected. We excluded those patients with incomplete availability of precontrast T1-weighted, postcontrast T1-weighted, FLAIR, T2-weighted, and DSC MRI sequences (requiring either three-dimensional acquisitions or two-dimensional with axial orientation; $n=287$ ); heavy motion artifacts $(n=14)$; insufficient contrast agent administration at DSC MRI or at postcontrast T1-weighted imaging $(n=31)$; or because of corrupt data after Digital Imaging and Communications in Medicine data format to the Neuroimaging Informatics Technology Initiative data format $(n=10)$ (Fig 1).

\section{MRI Examinations}

MRI examinations were performed at baseline and every 6 weeks until week 24, afterward every 3 months (13). Specifically, imaging with standardized imaging protocol was aligned with the consensus recommendations for a standardized brain 


\section{EORTC-26101 phase II trial \\ ( $n=159$ patients)}

EORTC-26101 phase III trial ( $n=437$ patients)

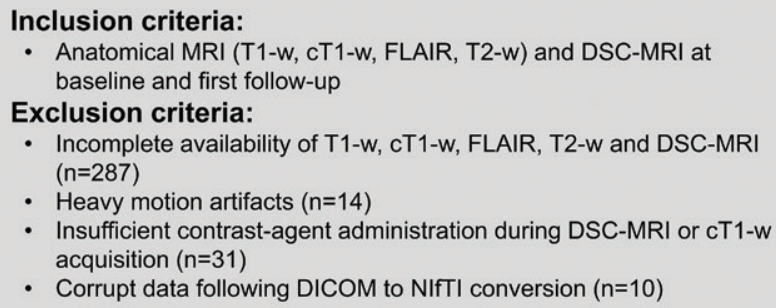

- Insufficient contrast-agent administration during DSC-MRI or cT1-w acquisition $(n=31)$

- Corrupt data following DICOM to NIfTI conversion $(n=10)$

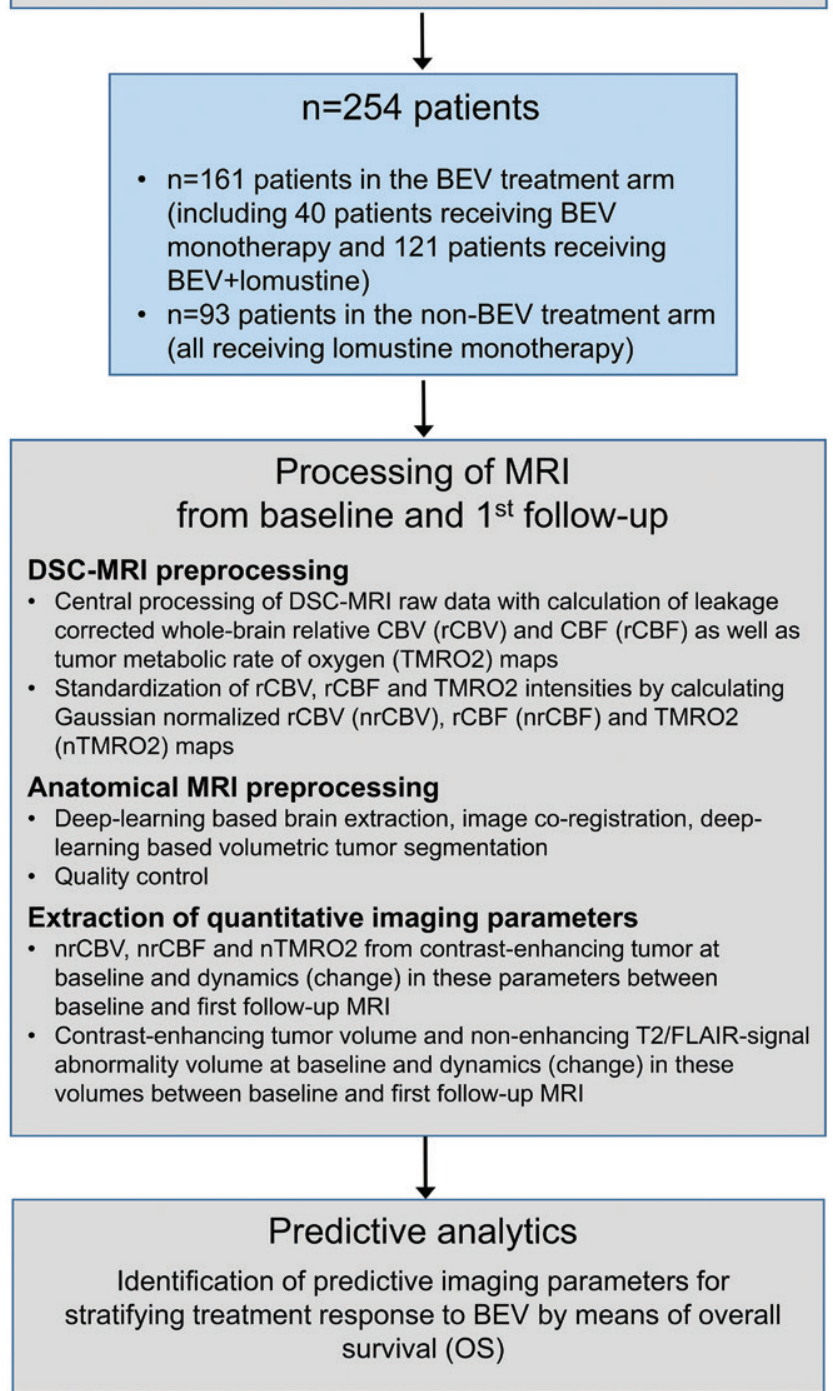

Figure 1: Flowchart of the procedures and analysis performed within the study. BEV = bevacizumab, cT 1-w = postcontrast T1-weighted, DSC = dynamic susceptibility contrast-enhanced imaging, DICOM = Digital Imaging and Communications in Medicine, EORTC = European Organization for Research and Treatment of Cancer, FLAIR = fluid-attenuated inversion recovery, $\mathrm{nrCBF}=$ Gaussian-normalized relative cerebral blood flow, $\mathrm{nrCBV}=$ Gaussian-normalized relative cerebral blood volume, nrTMRO2 = Gaussian-normalized tumor metabolic rate of oxygen, $\mathrm{Tl}-\mathrm{w}=$ precontrast $\mathrm{T} 1$ weighted, $\mathrm{T} 2-\mathrm{w}=\mathrm{T} 2$ weighted.

tumor imaging protocol in clinical trials (15) and included pre- and postcontrast T1-weighted, T2-weighted, and FLAIR images. In addition, optional DSC MRI was performed at the

discretion of the individual institution (sequence parameters listed in Appendix E1 [online]).

\section{MRI Postprocessing}

A flowchart depicting the analysis workflow is shown in Figure 1 with detailed description in Appendix E2 (online). MRI postprocessing was performed by G.B., a radiology resident with 5 years of experience in image processing, and subsequently checked by P.K., a board-certified radiologist with 7 years of experience. Specifically, processing of the anatomic sequences included automated deep learning-based brain extraction (16) followed by image registration and automated deep learningbased voxel-wise segmentation with volumetric quantification of the contrast-enhanced tumor volume and the noncontrast-enhanced T2 FLAIR signal abnormality volume as described previously $(14,16)$. All patients reported in our study were also included in previous works. However, there is no relevant overlap with our study, which analyzes the DSC MRI data and aims to identify prognostic and/or prospective imaging biomarkers.

Processing of DSC MRI sequences for calculating cerebral blood flow and cerebral blood volume maps was performed with dedicated software (Olea Sphere version 2.3, Olea Medical, La Ciotat, France). Specifically, whole-brain relative cerebral blood volume and relative cerebral blood flow maps were generated as previously described (17). Postprocessing of DSC MRI data for calculating tumor metabolic rate of oxygen maps was performed with Cercare Medical Neurosuite (Cercare Medical, Aarhus, Denmark), as previously described (18). Image intensity correction of the relative cerebral blood volume, relative cerebral blood flow, and tumor metabolic rate of oxygen maps was performed by calculating Gaussian-normalized relative cerebral blood volume (nrCBV), Gaussian-normalized relative cerebral blood flow (nrCBF), and Gaussiannormalized tumor metabolic rate of oxygen (nT$\mathrm{MRO}_{2}$ ) maps, as suggested by Ellingson et al (19). The median nrCBV, nrCBF, and nTMRO 2 values at baseline MRI and the dynamics in the nrCBV, nrCBF, and nTMRO 2 values between baseline and first follow-up MRI was calculated from the contrast-enhanced tumor volume.

\section{Outcomes}

The objective of our study was to identify predictive imaging biomarkers for stratifying treatment outcome (by means of OS) to bevacizumab therapy in patients with recurrent glioblastoma. OS was calculated from the date of randomization until death or last follow-up. Progression-free survival was calculated from the date of randomization until the date of tumor progression (as assessed by local Response Assessment 


\section{Table 1: Baseline Patient Characteristics}

\begin{tabular}{|c|c|c|c|}
\hline Parameter & BEV Group $(n=161)$ & Non-BEV Group $(n=93)$ & $P$ Value \\
\hline \multicolumn{4}{|l|}{ Clinical characteristics } \\
\hline \multicolumn{4}{|l|}{ Sex } \\
\hline No. of women & $64(40)$ & $35(38)$ & .84 \\
\hline No. of men & $97(60)$ & $58(62)$ & \\
\hline Median age $(y)^{*}$ & $58(51-64)$ & $60(51-64)$ & .73 \\
\hline \multicolumn{4}{|l|}{ WHO performance status } \\
\hline 0 & $54(34)$ & $33(35)$ & .86 \\
\hline$\geq 1$ & $107(66)$ & $60(65)$ & \\
\hline \multicolumn{4}{|l|}{ Use of glucocorticoids } \\
\hline No & $77(48)$ & $57(61)$ & .05 \\
\hline Yes & $84(52)$ & $36(39)$ & \\
\hline \multicolumn{4}{|l|}{ Sequence of treatment } \\
\hline BEV (at PD combination treatment with BEV and LOM) & $40(25)$ & $\ldots$ & NA \\
\hline Combination BEV and LOM (at PD investigator's choice) & $121(75)$ & $\ldots$ & \\
\hline LOM (at PD treatment with BEV) & $\ldots$ & $61(66)$ & \\
\hline LOM (at PD investigator’s choice) & $\ldots$ & $32(33)$ & \\
\hline \multicolumn{4}{|l|}{ Molecular characteristics } \\
\hline \multicolumn{4}{|l|}{ MGMT promoter methylation status } \\
\hline Methylated & $52(32)$ & $33(35)$ & .75 \\
\hline Unmethylated & $63(39)$ & $32(34)$ & \\
\hline Undetermined or missing data & $46(29)$ & $28(30)$ & \\
\hline \multicolumn{4}{|l|}{ GCIMP status } \\
\hline Positive & $3(1)$ & $2(2)$ & .99 \\
\hline Negative & $158(99)$ & $91(98)$ & \\
\hline \multicolumn{4}{|l|}{ Image acquisition characteristics } \\
\hline Median interval baseline MRI to randomization (d)* & $2(1-5)$ & $2(1-5)$ & .93 \\
\hline Median interval baseline MRI to first follow-up MRI (d)* & $46(42-50)$ & $45(42-49)$ & .16 \\
\hline \multicolumn{4}{|l|}{ Quantitative MRI parameters at baseline MRI } \\
\hline Median contrast-enhanced tumor volume $\left(\mathrm{cm}^{3}\right)^{*}$ & $13.77(6.09-30.28)$ & $15.04(7.36-30.46)$ & .69 \\
\hline Median noncontrast-enhanced T2-FLAIR signal abnormality volume $\left(\mathrm{cm}^{3}\right)^{*}$ & $63.63(35.08-106.09)$ & $62.38(30.09-98.55)$ & .94 \\
\hline Median nrCBV* & $1.00(0.68-1.37)$ & $0.99(0.68-1.42)$ & .78 \\
\hline Median nrCBF* & $1.03(0.74-1.40)$ & $1.04(0.69-1.46)$ & .71 \\
\hline Median nTMRO ${ }_{2}^{*}$ & $0.98(0.61-1.34)$ & $0.96(0.70-1.39)$ & .65 \\
\hline \multicolumn{4}{|c|}{$\begin{array}{l}\text { Note.-Unless otherwise specified, data are number of patients and data in parentheses are percentages. BEV = bevacizumab, FLAIR = flu- } \\
\text { id-attenuated inversion recovery, GCIMP = glioma-CpG island methylator phenotype, } \mathrm{LOM}=\text { lomustine, MGMT }=\mathrm{O} 6 \text {-methylguanine- } \\
\text { DNA methyltransferase, } \mathrm{NA}=\text { not assessed, nrCBF = Gaussian-normalized relative cerebral blood flow, nCBV }=\mathrm{Gaussian} \text {-normalized } \\
\text { relative cerebral blood volume, nTMRO }{ }_{2}=\text { Gaussian-normalized tumor metabolic rate of oxygen, PD = progressive disease, WHO = World } \\
\text { Health Organization. }\end{array}$} \\
\hline ata in parentheses & & & \\
\hline
\end{tabular}

in Neuro-oncology reading as part of the initial analysis of the EORTC 26101 trial [13]) or death, whichever occurred first.

\section{Statistical Analysis}

Statistical analysis was performed by using software ( $\mathrm{R}$ version 3.6.2; R Foundation for Statistical Computing, Vienna, Austria) by P.K. and T.G., a biostatistician with 21 years of experience. The differences in clinical, molecular, and extracted quantitative imaging parameters between the BEV and non$\mathrm{BEV}$ groups and between baseline and first follow-up MRI was assessed (Appendix E3 [online]).

The potential predictive association in terms of OS between the extracted imaging parameters (ie, contrast-enhanced tumor volumes; noncontrast-enhanced T2 FLAIR signal abnormality volumes; and median nrCBV, nrCBF, and nTMRO 2 values at baseline and the dynamics in all of these parameters between baseline and first follow-up MRI) and the effect of bevacizumab treatment was evaluated by constructing Cox proportional hazards regression models for each imaging parameter. Specifically, each model contained one imaging parameter, or continuous scale, as the putative biomarker; the treatment group (ie, BEV vs non-BEV); the treatment-by-biomarker interaction term to differentiate whether the putative biomarker is prognostic or predictive (20); and the sequence of treatment to rule out a confounding effect of different treatment regimens (ie, bevacizumab with lomustine at progression salvage treatment at the investigator's best choice vs bevacizumab alone at progression switch to bevacizumab with lomustine vs lomustine alone at progression 


\begin{tabular}{|c|c|c|c|}
\hline Parameter & BEV Group $(n=161)$ & Non-BEV Group $(n=93)$ & $P$ Value \\
\hline \multicolumn{4}{|c|}{ Contrast-enhanced tumor volume } \\
\hline Absolute change $\left(\mathrm{cm}^{3}\right)$ & $-7.40(-16.64$ to -2.22$)$ & $3.13(-3.19$ to 13.78$)$ & $<.001$ \\
\hline Relative change (\%) & $-66(-83$ to -35$)$ & $30(-17$ to 98$)$ & $<.001$ \\
\hline \multicolumn{4}{|c|}{ Noncontrast-enhanced T2 FLAIR signal abnormality volume } \\
\hline Absolute change $\left(\mathrm{cm}^{3}\right)$ & $-17.31(-57.91$ to -1.57$)$ & $4.76(-8.96$ to 35.33$)$ & $<.001$ \\
\hline Relative change (\%) & $-33(-71$ to -5$)$ & $10(-13$ to 82$)$ & $<.001$ \\
\hline \multicolumn{4}{|l|}{ nrCBV } \\
\hline Absolute change & $-0.14(-0.39$ to 0.12$)$ & $0.02(-0.15$ to 0.15$)$ & $<.001$ \\
\hline Relative change (\%) & $-16(-41$ to 13$)$ & $1(-12$ to 22$)$ & $<.001$ \\
\hline \multicolumn{4}{|l|}{$\mathrm{nrCBF}$} \\
\hline Absolute change & $-0.15(-0.37$ to 0.11$)$ & $0.01(-0.13$ to 0.15$)$ & $<.001$ \\
\hline Relative change (\%) & $-18(-36$ to 12$)$ & $1(-15$ to 19$)$ & $<.001$ \\
\hline \multicolumn{4}{|l|}{$\mathrm{nTMRO}_{2}$} \\
\hline Absolute change & $-0.14(-0.39$ to 0.16$)$ & $0(-0.12$ to 0.15$)$ & .004 \\
\hline Relative change (\%) & $-18(-39$ to 17$)$ & $0(-12$ to 18$)$ & .001 \\
\hline
\end{tabular}

Note.-Data in parentheses are interquartile range. $P$ values derived from a Wilcoxon rank sum test comparing the distribution of a given imaging parameter between the BEV and non-BEV group. BEV = bevacizumab, FLAIR = fluid-attenuated inversion recovery, $\mathrm{nrCBF}=$ Gaussian-normalized relative cerebral blood flow, nrCBV = Gaussian-normalized relative cerebral blood volume, $\mathrm{nTMRO}_{2}=\mathrm{Gaussian}-$ normalized tumor metabolic rate of oxygen.

switch to bevacizumab vs lomustine alone at progression salvage treatment at the investigator's best choice). A significant treatment-by-biomarker interaction term would indicate that the treatment effect differs by imaging parameter and thus would correspond to a predictive imaging biomarker (20). $P$ values from individual models were adjusted for multiple comparison by using the false discovery rate procedure.

Multivariable Cox proportional hazards regression modeling for OS was separately performed for both BEV and non-BEV groups to assess the independent significance of imaging parameters. The model was adjusted for potential clinical and molecular confounders (age, sex, World Health Organization performance status, glucocorticoid administration at baseline, O6-methylguanine-DNA methyltransferase promoter methylation status, and glioma-CpG island methylator phenotype status). $P$ values less than .05 were considered to indicate statistical significance.

\section{Results}

\section{Patient Characteristics}

Among 596 patients in the EORTC 26101 phase II and III study, a subset of 254 patients (42.6\%) met the criteria for this secondary analysis (Fig 1). These 254 patients included 161 patients $(63.4 \%)$ in the BEV group and 93 patients $(36.6 \%)$ in the non-BEV group. Baseline clinical, molecular, and image characteristics were equally distributed between the BEV and non-BEV groups (Table 1).

\section{Differences in Tumor Volumes, Angiogenesis, and Oxygenation Characteristics}

The extracted quantitative imaging parameters showed a significant change in response to treatment by assessing their dynamics between baseline MRI and first follow-up MRI, and the change in the BEV group differed significantly from the change in the non-BEV group $(P \leq .004$, each; Table 2 , Fig E1 [online]). Specifically, the contrast-enhanced tumor volumes decreased for patients in the BEV group (median change, $-66 \%$; interquartile range $[\mathrm{IQR}],-83 \%$ to $-35 \%$; $P<.001$ ), whereas they increased for patients in the non$\mathrm{BEV}$ group (median change between baseline MRI and first follow-up MRI, 30\%; IQR, $-17 \%$ to $98 \%$; $P=.001$; Fig 2, $A$, Table 2). Similarly, the unenhanced T2 and FLAIR signal abnormality volumes decreased for patients in the BEV group (median change between baseline MRI and first follow-up MRI, $-33 \%$; IQR, $-71 \%$ to $-5 \%$; $P<.001$ ), whereas they increased for patients in the non-BEV group (median change, $10 \%$; IQR, $-13 \%$ to $82 \% ; P=.02$; Fig 2 , $B$, Table 2 ).

The nrCBV, which reflects tumor angiogenesis, decreased in the BEV group (median change between baseline MRI and first follow-up MRI, $-16 \%$; IQR, $-41 \%$ to $13 \%$; $P<.001$ ) but not for the non-BEV group (median change between baseline MRI and first follow-up MRI, $1 \%$; IQR, $-12 \%$ to $22 \%$; $P=.79$ ) (Fig 3, $A$, Table 2 ). There was a close positive correlation between nrCBV and nrCBF values (which reflects intratumoral blood flow) as well as nTMRO, values (which reflects intratumoral oxygenation) both at baseline (nrCBF and nT$\mathrm{MRO}_{2}$ Pearson correlation coefficients, 0.99 [95\% confidence interval $\{\mathrm{CI}\}: 0.98,0.99 ; P<.001]$ and 0.97 [95\% CI: 0.96, 0.97; $P<.001]$, respectively) and in response to treatment (nrCBF and nTMRO 2 Pearson correlation coefficients, 0.97 [95\% CI: 0.96, 0.98; $P<.001]$ and 0.92 [95\% CI: 0.90, 0.94; $P<.001$ ], respectively; Figs E2, E3 [online]). Both nrCBF and nTMRO ${ }_{2}$ decreased for the BEV group (nrCBF vs nT$\mathrm{MRO}_{2}$ median change between baseline MRI and first followup MRI, $-18 \%$ [IQR, $-36 \%$ to $12 \% ; P<.001]$ vs $-18 \%$ [IQR, $-39 \%$ to $17 \% ; P=.001]$, respectively) but not for the non-BEV group ( $\mathrm{nrCBF}$ vs $\mathrm{nTMRO}$ median change between baseline MRI and first follow-up MRI, 1\% [IQR, $-15 \%$ to 

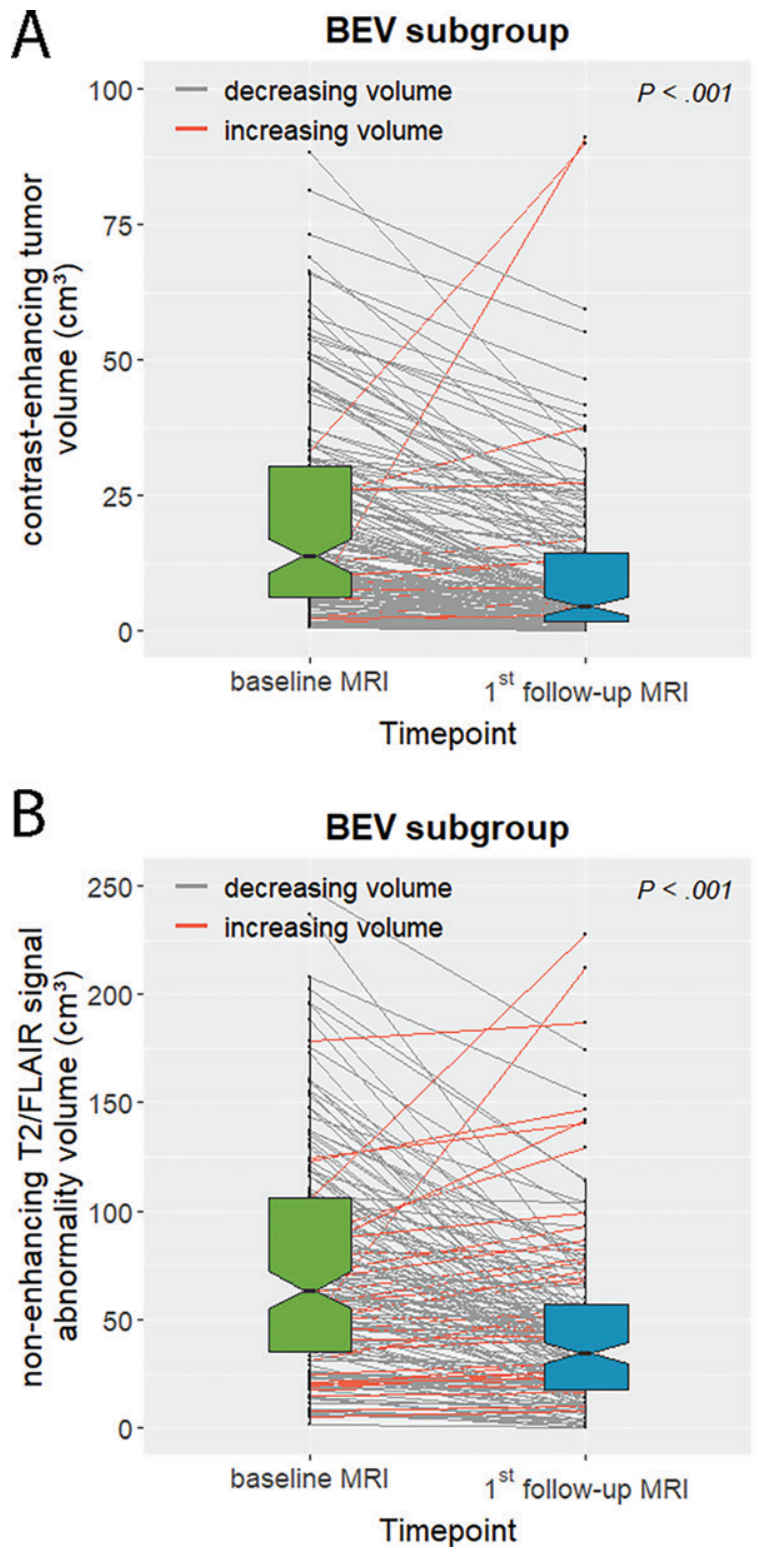

non-BEV subgroup

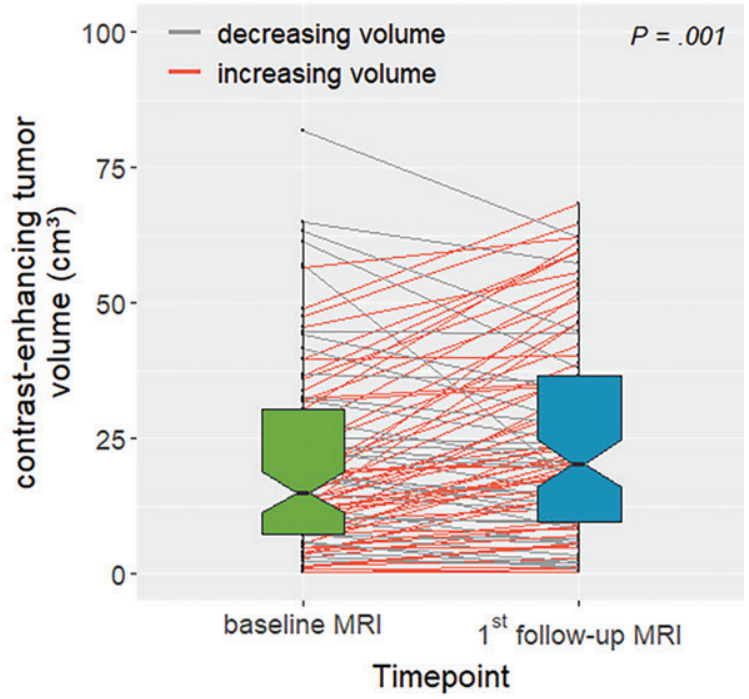

non-BEV subgroup

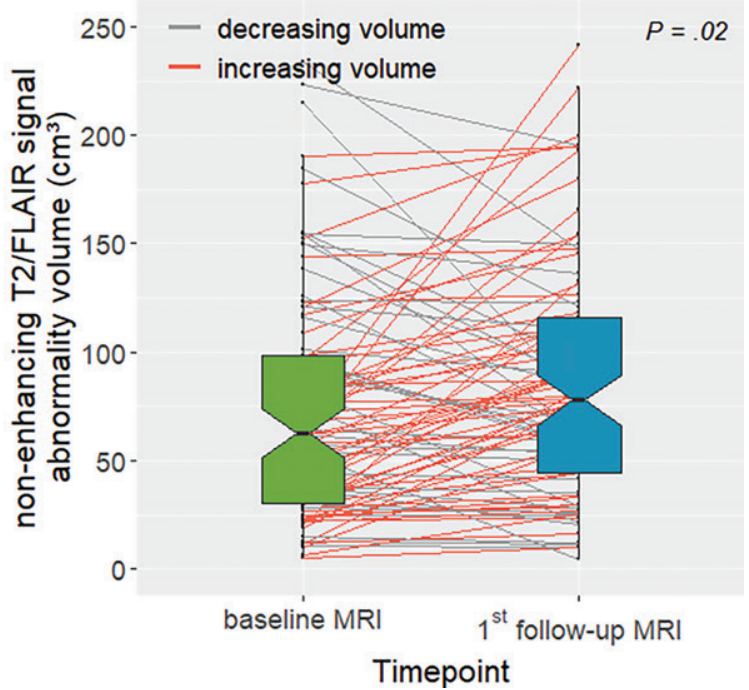

Figure 2: $A$, Contrast-enhanced tumor volumes and, $B$, noncontrast-enhanced T2 fluid-attenuated inversion recovery (FLAIR) signal abnormality volumes at baseline and first follow-up MRI and the dynamics in these volumes between the two points (increasing volume and decreasing volume) BEV = bevacizumab.

$19 \% ; P=.97]$ and $0 \%[\mathrm{IQR},-12 \%$ to $18 \% ; P=.64]$, respectively; Fig 3, $B, C$, Table 2).

\section{Progression-Free Survival and OS}

Progression-free survival was longer in the BEV group (3.7 months; 95\% CI: 3.0, 4.2) than in the non-BEV group $(2.5$ months; 95\% CI: $1.5,2.9)(P=.01)$, whereas OS was not different between the BEV group (8.9 months; 95\% CI: 7.7, 10.2 ) and the non-BEV group (9.8 months; $95 \% \mathrm{CI}: 8.6$, 12.1) $(P=.15$; Fig E4 [online]). A quantitative interaction between the extracted imaging parameters and the treatment variable was found for the dynamics in contrast-enhanced tumor volumes between baseline and first follow-up (Table 3). Specifically, the model's hazard ratio of 0.96 (95\% CI: 0.94, 0.98; false discovery rate-adjusted $P=.011$ ) for the interaction term between imaging parameter and treatment variable and the hazard ratio of 1.04 (95\% CI: $1.01,1.06 ; P$ $=.001$ ) for the imaging parameter indicated superior OS for those patients in the non-BEV subgroup with greater reduction in contrast-enhanced tumor volume (Fig E5 [online]). There was no predictive association in terms of OS between any of the extracted imaging parameters and the effect of bevacizumab treatment (false discovery rate-adjusted $P>$ .05 for the interaction term in each of the remaining models; Table 3).

Multivariable Cox proportional hazards regression adjusted for clinical and molecular parameters demonstrated an independent prognostic significance (in terms of OS) for the baseline contrast-enhanced tumor volume (BEV vs non-BEV group hazard ratio, 1.02 [95\% CI: $1.01,1.04 ; P=.01]$ and $1.03[95 \%$ CI: $1.01,1.06 ; P=.01$ ], respectively) (Figs 4, 5, $A$ ). Notably, the nrCBV dynamics between baseline and first follow-up MRI 


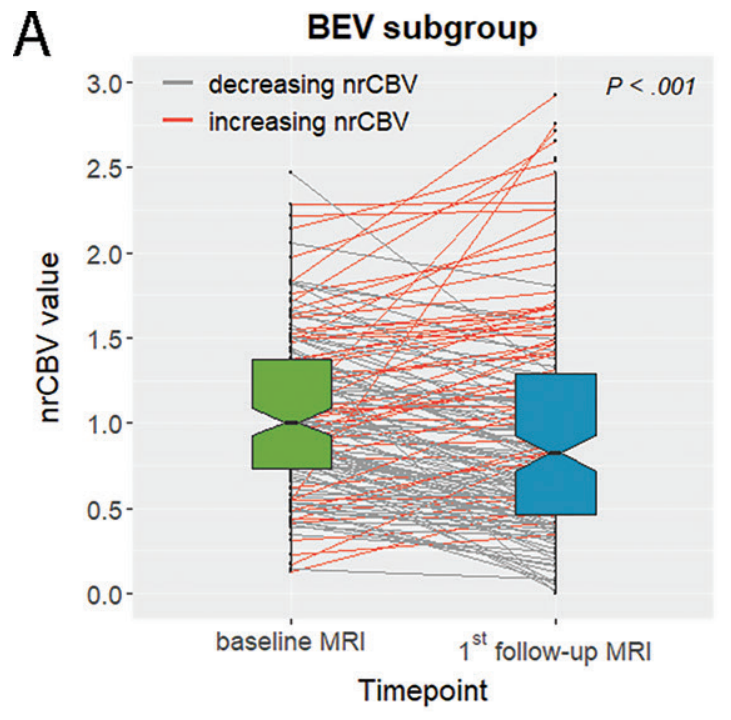

B

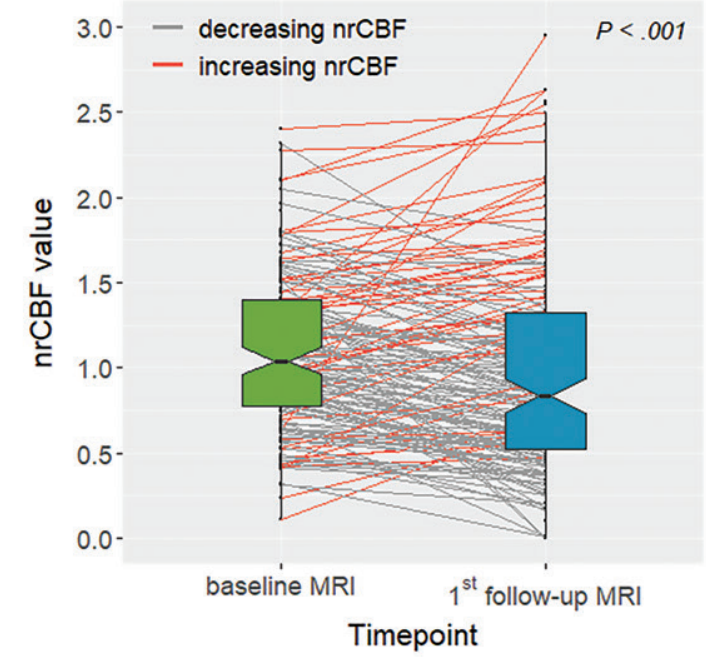

C

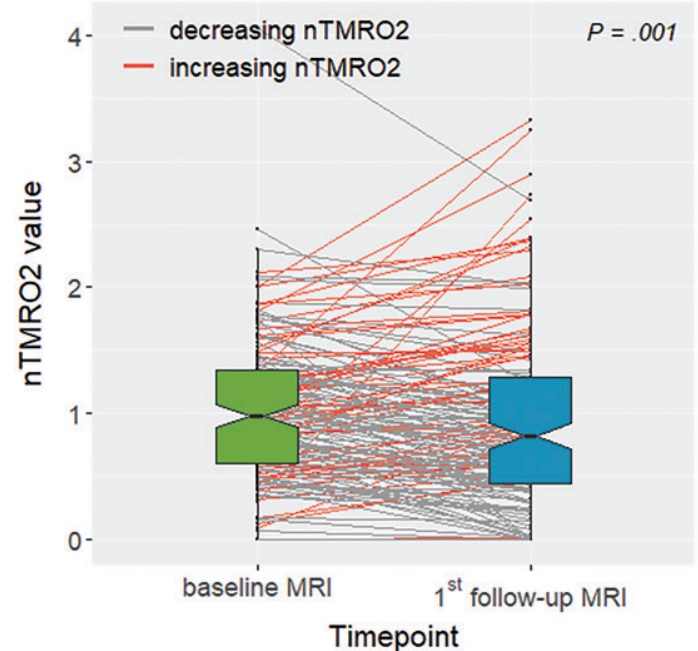

non-BEV subgroup
3.0- - decreasing nrCBV
- increasing nrCBV
$P=.79$

$2.5-$

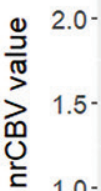

$0.5-$

$0.0-$

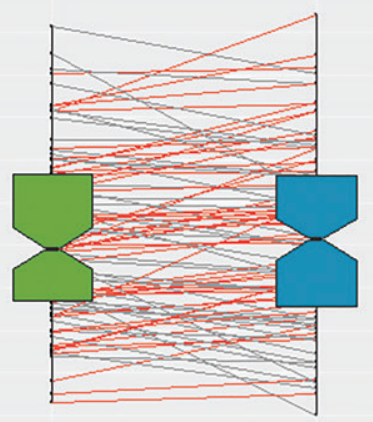

baseline MRI $\quad 1^{\text {st }}$ follow-up MRI
Timepoint

non-BEV subgroup
3.0- - decreasing $\mathrm{nrCBF} \longrightarrow P=.97$ - increasing nrCBF

2.5

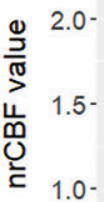

0.5

$0.0-$

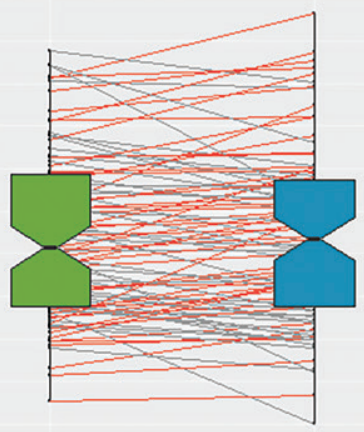

baseline MRI $\quad 1^{\text {st }}$ follow-up MRI Timepoint

non-BEV subgroup

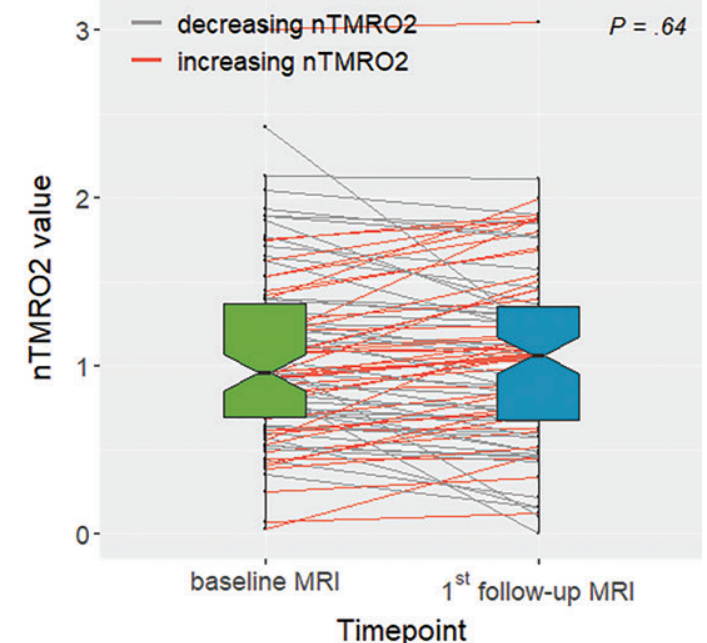

Timepoint

Figure 3: Distribution of, A, Gaussian-normalized relative cerebral blood volume (nrCBV), B, Gaussian-normalized relative cerebral blood flow (nrCBF), and, $C$, tumor metabolic rate of oxygen (nTMRO2) values at baseline and first follow-up MRI and the dynamics between these two points (increasing nrCBV, $\mathrm{nrCBF}$, or nTMRO2; or decreasing $\mathrm{nr} C B V$, $\mathrm{nrCBF}$ or $\mathrm{nTMRO} 2$ ). $\mathrm{BEV}=$ bevacizumab. 


\begin{tabular}{|c|c|c|c|}
\hline Parameter & Hazard Ratio & $P$ Value & FDR-adjusted $P$ Value \\
\hline \multicolumn{4}{|l|}{ Baseline MRI } \\
\hline nrCBV & $0.80(0.49,1.29)$ & .35 & .59 \\
\hline $\mathrm{nrCBF}$ & $0.77(0.46,1.28)$ & .31 & .59 \\
\hline $\mathrm{nTMRO}_{2}$ & $0.72(0.45,1.16)$ & .17 & .58 \\
\hline $\operatorname{CET}\left(\mathrm{cm}^{3}\right)$ & $1.01(0.99,1.02)$ & .36 & .59 \\
\hline $\mathrm{NE}\left(\mathrm{cm}^{3}\right)$ & $1.00(1.00,1.01)$ & .87 & .95 \\
\hline \multicolumn{4}{|c|}{ Change between baseline and first follow-up MRI } \\
\hline nrCBV & $1.08(0.40,2.91)$ & .88 & .95 \\
\hline $\mathrm{nrCBF}$ & $0.87(0.30,2.49)$ & .79 & .95 \\
\hline $\mathrm{nTMRO}_{2}$ & $1.03(0.42,2.50)$ & .95 & .95 \\
\hline $\operatorname{CET}\left(\mathrm{cm}^{3}\right)$ & $0.96(0.94,0.98)$ & .001 & $.011^{*}$ \\
\hline $\mathrm{NE}\left(\mathrm{cm}^{3}\right)$ & $0.99(0.99,1.00)$ & .04 & .18 \\
\hline \multicolumn{4}{|c|}{$\begin{array}{l}\text { Note.-Data in parentheses are } 95 \% \text { confidence intervals. CET }=\text { contrast-enhanced tumor volume, FDR }=\text { false discovery rate, } \mathrm{NE}=\text { non- } \\
\text { contrast enhanced } \mathrm{T} 2 \text { fluid-attenuated inversion recovery signal abnormality, nrCBF }=\text { Gaussian-normalized relative cerebral blood volume, } \\
\text { nrCBV }=\text { Gaussian-normalized relative cerebral blood volume, nTMRO } \mathrm{N}_{2}=\text { Gaussian-normalized tumor metabolic rate of oxygen. } \\
* \text { Quantitative interaction indicating benefit for the non-bevacizumab group (Fig E5 [online]). The given hazard ratios and } P \text { values are } \\
\text { from the models' interaction term (imaging parameter: treatment effect). }\end{array}$} \\
\hline
\end{tabular}

was an independent predictor of OS for the BEV group (hazard ratio, 2.10 ; 95\% CI: $1.38,3.19 ; P<.001$ ), but not for the nonBEV group (hazard ratio, 0.27 ; $95 \%$ CI: $0.06,1.35 ; P=.11$ ) (Fig 4). Despite this finding, there was no predictive association between nrCBV dynamics and the effect of bevacizumab treatment (Table 3). Specifically, OS curves between the BEV group with decreasing nrCBV and the non-BEV group, regardless of increasing or decreasing nrCBV, were similar (Fig 5, B). Only patients in the BEV group with an increase in nrCBV demonstrated a comparatively shorter OS (Fig 5, B).

\section{Discussion}

There remains an unmet clinical need for easily and ideally noninvasively accessible surrogate biomarkers able to delineate molecular activity and predict outcome to antiangiogenic treatment in patients with glioblastoma $(10,17,21)$. We aimed to address these needs by analyzing the dynamic susceptibility contrast-enhanced (DSC) MRI data from the prospective randomized controlled multicenter phase II and III European Organization for Research and Treatment of Cancer 26101 trial. We applied a robust postprocessing workflow including harmonization and central processing of DSC MRI data with longitudinal quantification of Gaussiannormalized relative cerebral blood volume (nrCBV, reflecting angiogenesis), Gaussian-normalized relative cerebral blood flow (nrCBF, reflecting blood flow), and tumor metabolic rate of oxygen (nTMRO ${ }_{2}$, reflecting oxygenation status). We found that treatment with bevacizumab significantly decreases contrast-enhanced and noncontrast-enhanced T2 fluid-attenuated inversion recovery (FLAIR) signal abnormality tumor volumes (by a median of $66 \%$ and $33 \%$; $P<$ .001 each) and intratumoral angiogenesis, blood flow, and oxygen consumption (by a median of $16 \%-18 \% ; P \leq .001$ each). This effect was confined to patients who were administered bevacizumab (hereafter, the BEV group), whereas pa- tients in the group who were not administered bevacizumab (hereafter, the non-BEV group) showed an increase in contrast-enhanced and noncontrast-enhanced T2 FLAIR signal abnormality tumor volumes but unchanged perfusion parameters ( $\mathrm{nrCBV}$, nrCBV, and nTMRO ${ }_{2}$ ) in response to treatment. Although these findings reflect the effectiveness of bevacizumab for extending progression-free survival, we found no predictive association in terms of overall survival (OS) between the effect of bevacizumab treatment and the assessed imaging parameters. In accordance with the literature, we found that the baseline contrast-enhanced tumor volume was an independent prognostic significance for stratifying OS (ie, in both BEV and non-BEV groups [14,22,23]). Moreover, we found superior OS rates for patients with a greater reduction in contrast-enhanced tumor volume in the non-BEV group (compared with the BEV group), which reflects the dilemma of bevacizumab treatment, which frequently causes a rapid decline in the contrast-enhancing tumor, although this may partly result from normalization of abnormally permeable vessels and not necessarily indicate a true antitumor effect (24). Consequently, identifying patient subsets that may benefit from bevacizumab in terms of OS remains challenging and the optimal setting for bevacizumab in the treatment of glioblastoma needs further clarification.

Noninvasive characterization of intratumoral angiogenesis on DSC MRI has gained substantial interest for studying the effects of antiangiogenic agents like bevacizumab (25). Specifically, previous uncontrolled retrospective studies demonstrated that angiogenesis-related parameters obtained from DSC MRI may correlate with treatment outcome and help identify subsets in which bevacizumab is most beneficial in terms of OS $(9,10,17,18,26,27)$. Specifically, lower baseline values and a greater reduction in angiogenesis-related parameters obtained at DSC MRI have been linked to a favorable OS for bevacizumab $(9,10,17,18,26,27)$. Despite these intriguing findings, the utility 


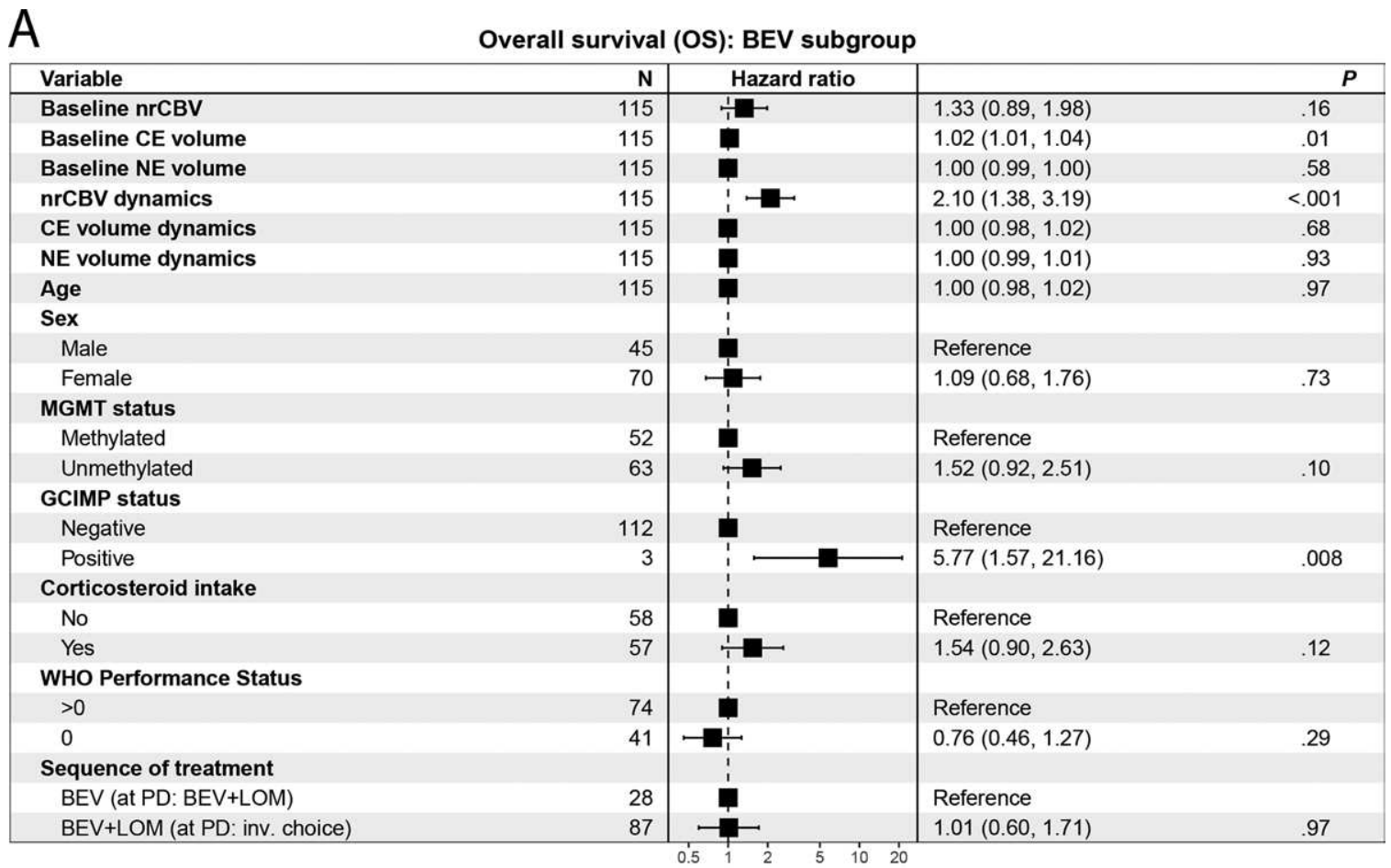

\section{B}

Overall survival (OS): non-BEV subgroup

\begin{tabular}{|c|c|c|c|c|}
\hline Variable & $\mathbf{N}$ & Hazard ratio & & $\mathrm{p}$ \\
\hline Baseline nrCBV & 65 & 7 & $1.28(0.71,2.34)$ & .41 \\
\hline Baseline CE volume & 65 & & $1.03(1.01,1.06)$ & .01 \\
\hline Baseline NE volume & 65 & & $1.00(0.99,1.01)$ & .56 \\
\hline nrCBV dynamics & 65 & - & $0.27(0.06,1.35)$ & .11 \\
\hline CE volume dynamics & 65 & & $1.05(1.02,1.08)$ & .002 \\
\hline NE volume dynamics & 65 & & $1.01(1.00,1.01)$ & .20 \\
\hline Age & 65 & & $1.02(0.99,1.05)$ & .19 \\
\hline \multicolumn{5}{|l|}{ Sex } \\
\hline Male & 30 & & Reference & \\
\hline Female & 35 & 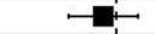 & $0.76(0.37,1.58)$ & .46 \\
\hline \multicolumn{5}{|l|}{ MGMT status } \\
\hline Methylated & 33 & & Reference & \\
\hline Unmethylated & 32 & 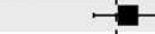 & $1.28(0.62,2.63)$ & .50 \\
\hline \multicolumn{5}{|l|}{ GCIMP status } \\
\hline Negative & 63 & & Reference & \\
\hline Positive & 2 & & $0.27(0.03,2.79)$ & .27 \\
\hline \multicolumn{5}{|l|}{ Corticosteroid intake } \\
\hline No & 39 & & Reference & \\
\hline Yes & 26 & & $2.75(1.13,6.69)$ & .003 \\
\hline \multicolumn{5}{|l|}{ WHO Performance Status } \\
\hline$>0$ & 41 & & Reference & \\
\hline 0 & 24 & 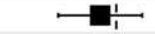 & $0.71(0.29,1.72)$ & .45 \\
\hline \multicolumn{5}{|l|}{ Sequence of treatment } \\
\hline LOM (at PD: BEV) & 43 & & Reference & \\
\hline LOM (at PD: inv. choice) & 22 & - & $0.78(0.41,1.47)$ & .44 \\
\hline
\end{tabular}

Figure 4: Multivariable Cox proportional hazards regression model for overall survival (OS) in, $A$, the BEV group and, $B$, the non-BEV group. BEV= bevacizumab, $\mathrm{CE}=$ contrast enhanced, $\mathrm{GCIMP}=$ glioma-CpG island methylator phenotype, $\mathrm{LOM}=$ lomustine, $\mathrm{MGMT}=\mathrm{O}$-methylguanine-DNA methyltransferase, $\mathrm{NE}=$ noncontrast enhanced, $\mathrm{nrCBV}=$ Gaussian-normalized relative cerebral blood volume, $\mathrm{PD}$ = progressive disease, $\mathrm{WHO}=$

World Health Organization.

of angiogenesis-related parameters obtained from DSC MRI as truly predictive imaging biomarkers has, to the knowledge of the authors, not been fully established because previous studies were based on the analysis of retrospective data sets $(10,17,18,26,27)$ or secondary analysis of uncontrolled phase II trials (9). Consequently, our results from the secondary analysis of the EORTC
26101 trial with an adequate control arm were necessary to reliable differentiate a truly predictive biomarker (which allows identification of the likelihood of sensitivity to bevacizumab treatment) from a prognostic biomarker (which reflects the patients overall outcome, regardless of therapy) (20). Moreover, several studies indicated that bevacizumab treatment restores 

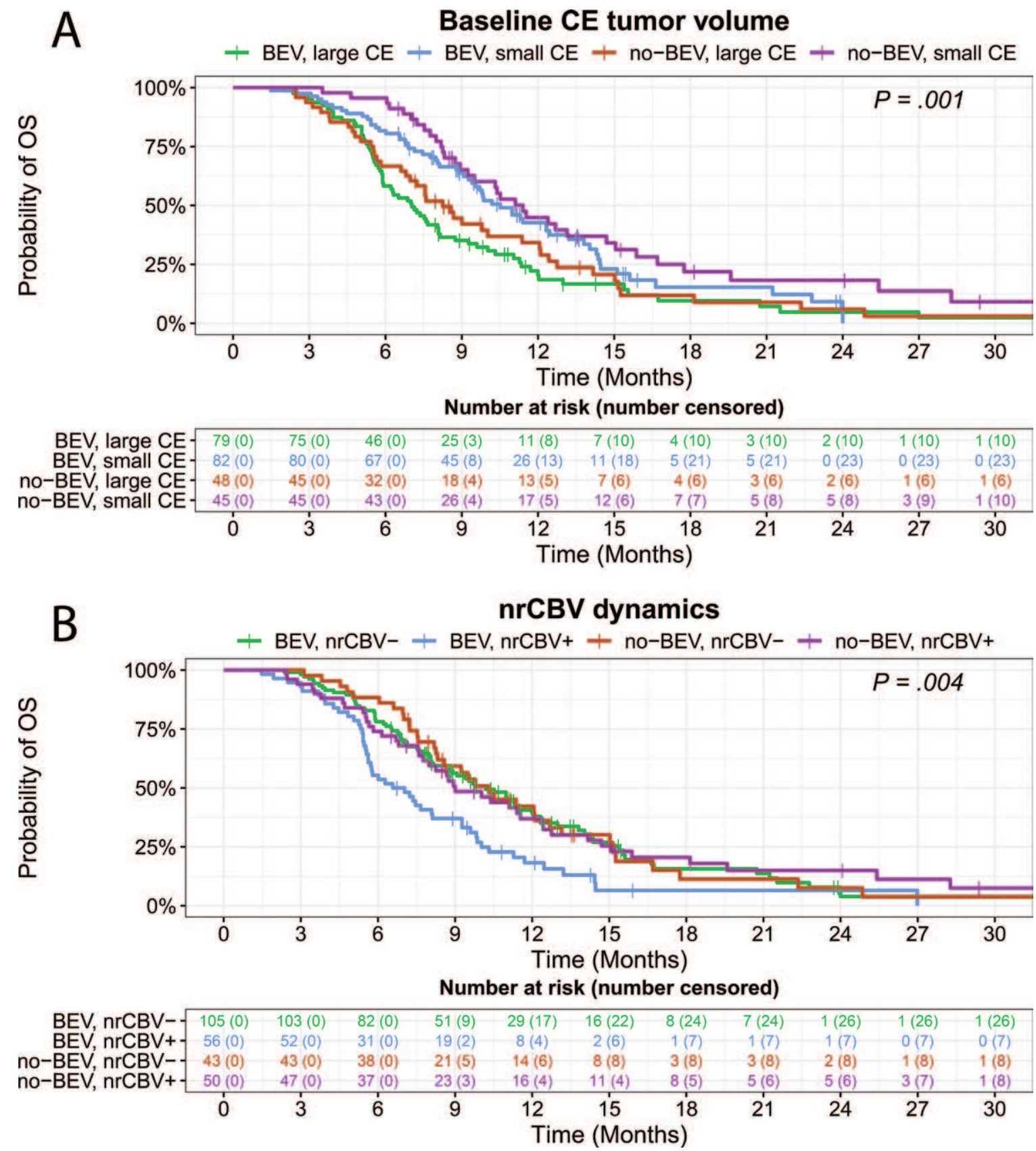

Figure 5: Kaplan-Meier plots for overall survival (OS) comparing outcome for the bevacizumab (BEV) versus non-BEV groups in terms of, $A$, contrast-enhanced (CE) tumor volume at baseline MRI and, B, change in Gaussian-normalized relative cerebral blood volume (nrCBV) between baseline and first follow-up MRI. BEV = bevacizumab, CE- = tumor volume smaller than the median contrast-enhanced tumor volume at baseline, $\mathrm{CE}+=$ tumor volume larger than the median contrast-enhanced tumor volume at baseline, $\mathrm{nrCBV}-=$ decreasing $\mathrm{nrCBV}$ between baseline and first follow-up MRI, nrCBV+ = increasing nrCBV between baseline and first follow-up MRI.

the abnormal structure and function of the tumor vasculature toward a more normal state and tumor blood flow and oxygenation transiently increases, thus providing an opportunity to improve the sensitivity toward radiation therapy and/or chemotherapy (28). The effect of bevacizumab on tumor oxygenation remains controversial, with evidence supporting either vascular regression, which is associated with increased intratumoral hypoxia $(29,30)$, or a so-called normalization of the tumor vasculature, resulting in improved tumor oxygenation and cytotoxic chemotherapy sensitivity $(31,32)$. We did not observe vascular normalization because of bevacizumab treatment, which is hypothesized to be advantageous for treatment outcome $(31,32)$. Instead we saw a positive linear association between $\mathrm{nrCBV}$, $\mathrm{nrCBF}$, and $\mathrm{nTMRO}$, which suggested that a reduction in tumor angiogenesis was also associated with a reduction in tumor blood flow and tumor oxygenation.

Our study had limitations. First, only a subset of 254 of 596 patients $(42.6 \%)$ met the criteria for this secondary analysis, with sample size determined by the availability of MRI data and not derived from a power calculation. Second, the high crossover 
rate of $66 \%$ (61 of 93 patients) in the non-BEV group who underwent bevacizumab treatment following disease progression may have complicated the identification of predictive imaging biomarkers for bevacizumab. However, multivariable Cox regression modeling demonstrated that the presence of crossover (sequence of treatment) was not a relevant confounder. Third, the randomization ratio in the EORTC 26101 trial led to an unbalanced distribution of patients in the BEV and non-BEV treatment arm. This resulted in a lower number of patients in the non-BEV treatment arm and could have reduced statistical power. Fourth, the follow-up measurements in our study (6-7 weeks after treatment initiation) may have been outside of the window during which vascular normalization occurs. Specifically, vascular normalization and improved tumor oxygenation is believed to occur from a few days until 4-5 weeks after initiation of bevacizumab treatment, whereas the follow-up measurement in our study may already fall within the phase of microvascular rarefaction and thus may have hampered the assessment of the vascular normalization theory (31).

In conclusion, secondary analysis of the European Organization for Research and Treatment of Cancer 26101 trial demonstrates that bevacizumab decreases tumor volumes, intratumoral angiogenesis, and oxygen consumption, thereby reflecting its effectiveness for extending progression-free survival. However, neither the suggested vascular normalization theory nor the value of assessing the extent of angiogenesis or oxygen consumption at dynamic susceptibility MRI as potential predictive biomarkers for stratifying overall survival in patients treated with bevacizumab could be substantiated.

Author contributions: Guarantor of integrity of entire study, P.K.; study concepts/ study design or data acquisition or data analysis/interpretation, all authors; manuscript drafting or manuscript revision for important intellectual content, all authors; approval of final version of submitted manuscript, all authors; agrees to ensure any questions related to the work are appropriately resolved, all authors; literature research, P.K., F.I., A.W., M.B.; clinical studies, P.K., I.P., M.S., U.N., T.K., A.W., M.W., M.P., A.v.D., M.B.; experimental studies, P.K., M.N., M.S., F.I., F.S., A.W., A.v.D.; statistical analysis, P.K., A.W., K.H.M.H., T.G.; and manuscript editing, P.K., M.B.H., M.N., M.S., M.F., U.N., T.K., A.W., S.H., M.W., M.P., A.v.D., K.H.M.H., L.Ø., T.G., W.W., M.B.

Disclosures of Conflicts of Interest: P.K. disclosed no relevant relationships. G.B. disclosed no relevant relationships. M.B.H. Activities related to the present article: disclosed no relevant relationships. Activities not related to the present article: disclosed money paid to author for employment from Cercare Medical; money from stock/stock options from Cercare Medical; patent pending regarding technology for computing the microvascular markers with Aarhus University. Other relationships: disclosed no relevant relationships. M.N. disclosed no relevant relationships. I.P. disclosed no relevant relationships. M.S. disclosed no relevant relationships. F.I. disclosed no relevant relationships. M.F. disclosed no relevant relationships. U.N. disclosed no relevant relationships. T.K. disclosed no relevant relationships. F.S. Activities related to the present article: disclosed no relevant relationships. Activities not related to the present article: disclosed money paid to author for consultancy from Abbvie; disclosed payment for lectures from Illumina and Medac. Other relationships: disclosed no relevant relationships. A.W. disclosed no relevant relationships. S.H. disclosed no relevant relationships. M.W. Activities related to the present article: disclosed no relevant relationships. Activities not related to the present article: disclosed consultancies from Abbvie, Basilea, Bristol Meyers Squibb, Celgene, Medac, Merck, Sharp and Dohme, Merck (EMD), Nerviano Medical Sciences, Orbus, Roche, and Tocagen; disclosed money to author's institution for grants from Abbvie, Adastra, Dracen, Merck, Sharp and Dohme (MSD), Merck (EMD), and Novocure. Other relationships: disclosed no relevant relationships. M.P. Activities related to the present article: disclosed no relevant relationships. Activities not related to the present article: disclosed money paid to author for consultancies from Apogenix and Bayer; disclosed grants/grants pending from Pfizer, Roche, and Bayer; disclosed money paid to author for royal- ties from Bayer; disclosed payment for educational presentations from $\mathrm{MGH}$ and MSKCC; disclosed money paid to author for stock/stock options from Biogen. Other relationships: disclosed no relevant relationships. A.v.D. disclosed no relevant relationships. K.H.M.H. disclosed no relevant relationships. L.Ø. Activities related to the present article: disclosed no relevant relationships. Activities not related to the present article: disclosed stock/stock options from Cercare. Other relationships: disclosed no relevant relationships. M.J.v.d.B. disclosed no relevant relationships. T.G. disclosed no relevant relationships. W.W. disclosed no relevant relationships. M.B. Activities related to the present article: disclosed no relevant relationships. Activities not related to the present article: disclosed money paid to author for board membership from Vascular Dynamics, Braun, and Boehringer; disclosed grants/grants pending from the European Union, DFG, Hopp Foundation, Novartis, Guerbet, Stryker, and Siemens; disclosed money paid to author for lectures from Novartis, Teva, Grifols, Bayer, and Merck. Other relationships: disclosed no relevant relationships.

\section{References}

1. Friedman HS, Prados MD, Wen PY, et al. Bevacizumab alone and in combination with irinotecan in recurrent glioblastoma. J Clin Oncol 2009;27(28):4733-4740.

2. Kreisl TN, Kim L, Moore K, et al. Phase II trial of single-agent bevacizumab followed by bevacizumab plus irinotecan at tumor progression in recurrent glioblastoma. J Clin Oncol 2009;27(5):740-745.

3. Chinot OL, Wick W, Mason W, et al. Bevacizumab plus radiotherapy-temozolomide for newly diagnosed glioblastoma. N Engl J Med 2014;370(8):709-722.

4. Gilbert MR, Dignam JJ, Armstrong TS, et al. A randomized trial of bevacizumab for newly diagnosed glioblastoma. N Engl J Med 2014;370(8):699-708.

5. Wick W, Brandes A, Gorlia T, et al. Phase III trial exploring the combination of bevacizumab and lomustine in patients with first recurrence of a glioblastoma: the EORTC 26101 trial. 2015 SNO Annual Meeting, San Antonio, 2015.

6. Wick W, Osswald M, Wick A, Winkler F. Treatment of glioblastoma in adults. Ther Adv Neurol Disord 2018;11:1756286418790452.

7. Sandmann T, Bourgon R, Garcia J, et al. Patients With Proneural Glioblastoma May Derive Overall Survival Benefit From the Addition of Bevacizumab to First-Line Radiotherapy and Temozolomide: Retrospective Analysis of the AVAglio Trial. J Clin Oncol 2015;33(25):2735-2744.

8. Mayer TM. Can We Predict Bevacizumab Responders in Patients With Glioblastoma? J Clin Oncol 2015;33(25):2721-2722.

9. Schmainda KM, Zhang Z, Prah M, et al. Dynamic susceptibility contrast MRI measures of relative cerebral blood volume as a prognostic marker for overall survival in recurrent glioblastoma: results from the ACRIN 6677/RTOG 0625 multicenter trial. Neuro Oncol 2015;17(8):1148-1156.

10. Kickingereder P, Wiestler B, Burth S, et al. Relative cerebral blood volume is a potential predictive imaging biomarker of bevacizumab efficacy in recurrent glioblastoma. Neuro Oncol 2015;17(8):1139-1147.

11. Sorensen AG, Batchelor TT, Zhang WT, et al. A "vascular normalization index" as potential mechanistic biomarker to predict survival after a single dose of cediranib in recurrent glioblastoma patients. Cancer Res 2009;69(13):5296-5300.

12. Wick W, Stupp R, Gorlia T, et al. Phase II part of EORTC study 26101: The sequence of bevacizumab and lomustine in patients with first recurrence of a glioblastoma. J Clin Oncol 2016;34(15 suppl):2019.

13. Wick W, Gorlia T, Bendszus M, et al. Lomustine and Bevacizumab in Progressive Glioblastoma. N Engl J Med 2017;377(20):1954-1963.

14. Kickingereder P, Isensee F, Tursunova I, et al. Automated quantitative tumour response assessment of MRI in neuro-oncology with artificial neural networks: a multicentre, retrospective study. Lancet Oncol 2019;20(5):728-740.

15. Ellingson BM, Bendszus M, Boxerman J, et al. Consensus recommendations for a standardized Brain Tumor Imaging Protocol in clinical trials. Neuro Oncol 2015;17(9): 1188-1198.

16. Isensee F, Schell M, Tursunova I, et al. Automated brain extraction of multisequence MRI using artifi cial neural networks. Hum Brain Mapp 2019;40(17):4952-4964.

17. Kickingereder P, Radbruch A, Burth S, et al. MR Perfusion-derived Hemodynamic Parametric Response Mapping of Bevacizumab Efficacy in Recurrent Glioblastoma. Radiology 2016;279(2):542-552.

18. Bonekamp D, Mouridsen K, Radbruch A, et al. Assessment of tumor oxygenation and its impact on treatment response in bevacizumab-treated recurrent glioblastoma. J Cereb Blood Flow Metab 2017;37(2):485-494.

19. Ellingson BM, Zaw T, Cloughesy TF, et al. Comparison between intensity normalization techniques for dynamic susceptibility contrast (DSC)-MRI estimates of cerebral blood volume (CBV) in human gliomas. J Magn Reson Imaging 2012;35(6):14721477.

20. Ballman KV. Biomarker: Predictive or Prognostic? J Clin Oncol 2015;33(33):39683971.

21. Nowosielski M, Wiestler B, Goebel G, et al. Progression types after antiangiogenic therapy are related to outcome in recurrent glioblastoma. Neurology 2014;82(19): 1684-1692.

22. Ellingson BM, Harris RJ, Woodworth DC, et al. Baseline pretreatment contrast enhancing tumor volume including central necrosis is a prognostic factor in recurrent glioblastoma: evidence from single and multicenter trials. Neuro Oncol 2017;19(1):89-98.

23. Ellingson BM, Abrey LE, Nelson SJ, et al. Validation of postoperative residual contrast-enhancing tumor volume as an independent prognostic factor for overall survival in newly diagnosed glioblastoma. Neuro Oncol 2018;20(9):1240-1250.

24. Wen PY, Macdonald DR, Reardon DA, et al. Updated response assessment criteria for high-grade gliomas: response assessment in neuro-oncology working group. J Clin Oncol 2010;28(11):1963-1972. 
25. Kickingereder P, Park JE, Boxerman JL. Advanced Physiologic Imaging: Perfusion Theory and Applications. In: Pope WB, ed. Glioma Imaging: Physiologic, Metabolic, and Molecular Approaches. Cham, Switzerland: Springer International, 2020; 61-91.

26. Schmainda KM, Prah M, Connelly J, et al. Dynamic-susceptibility contrast agent MRI measures of relative cerebral blood volume predict response to bevacizumab in recurrent high-grade glioma. Neuro Oncol 2014;16(6):880-888.

27. Verhoeff JJ, Lavini C, van Linde ME, et al. Bevacizumab and dose-intense temozolomide in recurrent high-grade glioma. Ann Oncol 2010;21(8):1723-1727.

28. Li W, Quan YY, Li Y, Lu L, Cui M. Monitoring of tumor vascular normalization: the key points from basic research to clinical application. Cancer Manag Res 2018;10:41634172 .
29. Xu H, Rahimpour S, Nesvick CL, et al. Activation of hypoxia signaling induces phenotypic transformation of glioma cells: implications for bevacizumab antiangiogenic therapy. Oncotarget 2015;6(14):11882-11893.

30. Kerbel R, Folkman J. Clinical translation of angiogenesis inhibitors. Nat Rev Cancer 2002;2(10):727-739.

31. Jain RK. Normalizing tumor microenvironment to treat cancer: bench to bedside to biomarkers. J Clin Oncol 2013;31(17):2205-2218.

32. Batchelor TT, Gerstner ER, Emblem KE, et al. Improved tumor oxygenation and survival in glioblastoma patients who show increased blood perfusion after cediranib and chemoradiation. Proc Natl Acad Sci U S A 2013;110(47):19059-19064. 